\title{
Male Lower Urinary Tract Symptoms and Cardiovascular Events: A Systematic Review and Meta-analysis
}

\author{
Mauro Gacci $^{a, *}$, Giovanni Corona $^{b}$, Arcangelo Sebastianelli $^{a}$, Sergio Serni $^{a}$, Cosimo De Nunzio $^{c}$, \\ Mario Maggi ${ }^{d}$, Linda Vignozzi ${ }^{d}$, Giacomo Novara ${ }^{e}$, Kevin T. McVary ${ }^{f}$, Steven A. Kaplan ${ }^{g}$, \\ Stavros Gravas ${ }^{h}$, Christopher Chapple ${ }^{i}$ \\ ${ }^{a}$ Department of Urology, University of Florence, Careggi Hospital, Florence, Italy; ${ }^{\mathrm{b}}$ Endocrinology Unit, Maggiore-Bellaria Hospital, Bologna, Italy; \\ ' Department of Urology, Sant'Andrea Hospital, University “La Sapienza”, Rome, Italy; ${ }^{\mathrm{d}}$ Andrology Unit, University of Florence, Careggi Hospital, Florence, \\ Italy; ${ }^{\mathrm{e}}$ Department of Surgery, Oncology, and Gastroenterology, Urology Clinic, University of Padua, Padua, Italy; ${ }^{\mathrm{f}}$ Division of Urology, Southern Illinois \\ University School of Medicine, Springfield, IL, USA; ${ }^{\mathrm{g}}$ Department of Urology, Mount Sinai Hospital, New York, NY, USA; ${ }^{\mathrm{h}}$ Department of Urology, University \\ Hospital of Larissa, Larissa, Greece; ' Department of Urology, Sheffield Teaching Hospitals NHS Trust, Sheffield, UK
}

Article history:

Associate Editor:

Christian Gratzke

\section{Keywords:}

Prostate

Benign prostatic enlargement

Lower urinary tract symptoms

Major cardiac adverse event

\section{Article info}

Accepted July 4, 2016

Benign prostatic hyperplasia

\begin{abstract}
Context: The correlation among metabolic syndrome, lower urinary tract symptoms (LUTS), and cardiovascular disease (CVD) is well established. In particular, CVD has been proposed as a potential risk factor for both LUTS progression and severity.

Objective: To evaluate whether LUTS severity can be considered as a significant risk factor of major adverse cardiac events (MACE) in the male population.

Evidence acquisition: A systematic literature search was performed using PubMed, Google Scholar, and Scopus. The combination of the following keywords was adopted in a free-text strategy: benign prostatic hyperplasia (BPH) or lower urinary tract symptoms (LUTS) and cardiovascular, cardio, major adverse cardiac events, MACE, heart disease, heart, myocardial infarction, myocardial, infarction, stroke, ischemic events, ischemic, cardiac death, coronary syndrome. We included all cross-sectional and longitudinal trials enrolling men and comparing the prevalence or incidence of MACE in men with moderate to severe LUTS compared with those without LUTS or with mild LUTS. The studies in which only nocturia was evaluated were excluded from the analysis.

Evidence synthesis: Of 477 retrieved articles, 5 trials longitudinally reported the incidence of MACE in patients with moderate to severe LUTS in comparisons to those with mild or no LUTS and 10 studies reported the prevalence of history of MACE at enrollment. All were included in the present meta-analysis. Among cross-sectional studies, 38218 patients and 2527 MACE were included in the meta-analysis. The mean age of enrolled patients was $62.2 \pm 8.0 \mathrm{yr}$. Presence of moderate to severe LUTS significantly increased the risk of reported history of MACE $(p<0.001)$. Metaregression analyses showed that the risk of MACE was lower in older patients and higher in those with diabetes. The association between LUTS-related MACE and diabetes was confirmed in a multivariate regression model after adjusting for age (adjusted $r=0.498 ; p<0.0001$ ). Longitudinal trials included 25494 patients and 2291 MACE. The mean age of enrolled patients was $52.5 \pm 5.5 \mathrm{yr}$, and mean follow-up was $86.8 \pm 22.1 \mathrm{mo}$. Presence of moderate to severe LUTS was associated with an increased incidence of MACE compared with the rest of the sample (odds ratio: 1.68; 95\% confidence interval, 1.13-2.50; $p=0.01$ ).
\end{abstract}

* Corresponding author. Department of Urology, University of Florence, Viale A. Gramsci 7, Florence, 50121, Italy.

E-mail address: maurogacci@yahoo.it (M. Gacci).

http://dx.doi.org/10.1016/j.eururo.2016.07.007

0302-2838/C 2016 European Association of Urology. Published by Elsevier B.V. All rights reserved. 
Conclusions: Men with moderate to severe LUTS seem to have an increased risk of MACE. A holistic approach in considering the morbidities of aging men should be strongly encouraged and represents an important role for the practicing urologist.

Patient summary: We evaluated whether the severity of lower urinary tract symptoms could be considered as a significant risk factor for major adverse cardiac events (MACE) in the male population. We demonstrated that men with moderate to severe LUTS have an increased risk of MACE.

(C) 2016 European Association of Urology. Published by Elsevier B.V. All rights reserved.

\section{Introduction}

Lower urinary tract symptoms (LUTS) due to benign prostatic hyperplasia (BPH; LUTS/BPH) is one of the most frequently occurring urologic diseases in elderly men. Specifically, the incidence of moderate to severe LUTS in men aged $>50 \mathrm{yr}$ is $30-40 \%$ [1], with an increasing linear prevalence of LUTS with age [2]. Moreover, because of the global aging of Western populations, the cost burden associated with the management of LUTS will increase substantially over the next decades [3].

A noteworthy amount of preclinical and clinical evidence suggests a possible association among metabolic factors, metabolic syndrome (MetS), and male LUTS [4-6]. In particular, triglyceride and cholesterol levels seem to have a detrimental effect on prostatic cells, boosting prostate inflammation, which can be associated with the development and progression of LUTS/BPH [5]. Moreover, a recent meta-analysis demonstrated that obesity, dyslipidemia, and age are significant risk factors of having MetS as a determinant of benign prostate enlargement (BPE) [6]; therefore, LUTS seems to share a number of risk factors with cardiovascular diseases (CVDs). Accordingly, the European Association of Urology guidelines recognize the need to investigate these relevant comorbidities, including diseases, medications, and lifestyles [4]. Similarly, insulin resistance, dyslipidemia, and hypertension have been proven to be strong determinants of the development of CVD. In 2005, the American Diabetes Association and the European Association for the Study of Diabetes emphasized that each risk factor can cluster with others, leading to a single pathophysiologic conditions, defined as MetS [7]. In a recent metaanalysis on the cardiovascular risk associated with the MetS, including data from 87 studies and 951083 patients, MetS was associated with a twofold increase in risk of CVD, CVD mortality, myocardial infarction, and stroke and a 1.5-fold increase in risk of all-cause mortality [8].

Because correlations between MetS and both LUTS and CVD have been proven, CVD has been proposed as a potential risk factor for LUTS progression. In addition, LUTS severity has been investigated as an early clinical manifestation of a larger subclinical systemic disorder that can progress in severe CVD.

Specifically, the Massachusetts Male Aging Study (MMAS), a population-based study of 1709 noninstitutionalized randomly selected men aged 40-70 yr, demonstrated that coronary heart disease can independently increase risk for development of clinical BPH over $9 \mathrm{yr}$ [9]. In a recent crosssectional longitudinal study investigating the association between LUTS and both CVD and stroke in 2092 men aged $47 \mathrm{yr}$ and followed for an average of $6 \mathrm{yr}$, Wehrberger et al reported that men with severe LUTS (International Prostate Symptom Score $>20$ ) are at increased risk of CVD and stroke (odds ratios [ORs] of 1.28 and 1.66, respectively) [10].

Several publications have corroborated evidence that CVD could be a risk factor for worsening LUTS, whereas other prospective trials have suggested that severe LUTS could be associated with a modest but significant risk of developing a CVD. The aim of the present systematic review is to investigate the correlation between LUTS severity and risk of major adverse cardiac events (MACE), defined as angina pectoris, acute myocardial infarction, other chronic ischemic heart disease, transient ischemic attack, or cerebrovascular accident.

\section{Evidence acquisition}

This meta-analysis was performed according to the Metaanalysis Of Observational Studies in Epidemiology (MOOSE) Guidelines for Meta-Analyses and Systematic Reviews of Observational Studies [11]. Moreover, a Preferred Reporting Items for Systematic Reviews and Meta-Analyses checklist has been included as Supplementary Table 1 .

\subsection{Eligibility criteria}

All studies that specifically evaluated the presence of MACE in patients with or without moderate or severe LUTS were included in the analysis. The following inclusion criteria were adopted: (1) The study reported original data and was published in a peer-reviewed journal (ie, not a meeting abstract or review article), (2) the study was a cohort study (prospective or historical cohort) consisting of male human adults or the male cohort data could be extrapolated from the paper, and (3) the authors reported the incidence or the prevalence of cardiovascular events in patients with and without LUTS.

For the definition of MACE, we used the International Classification of Primary Care (ICPC) definitions for CVDs, including $\mathrm{K} 74$, angina pectoris; $\mathrm{K} 75$, acute myocardial infarction; K76, other chronic ischemic heart disease; K77, congestive heart failure; K89, transient ischemic attack; and K90, cerebrovascular accident [12].

\subsection{Information source and search strategy}

An extensive search of Medline, Embase, and Cochrane was performed and included the following words: benign 
Table 1 - Characteristics of the included studies.

\begin{tabular}{|c|c|c|c|c|c|c|c|c|c|c|}
\hline Study & Study design & $n$ & $\begin{array}{l}\text { Age, } \\
\text { mean }\end{array}$ & $\begin{array}{l}\text { Follow-up, } \\
\text { mo }\end{array}$ & $\begin{array}{l}\text { Patients with } \\
\text { moderate to } \\
\text { severe LUTS }\end{array}$ & $\begin{array}{l}\text { MACE in patients } \\
\text { with moderate to } \\
\text { severe LUTS, \% }\end{array}$ & $\begin{array}{l}\text { Patients with } \\
\text { mild or no LUTS }\end{array}$ & $\begin{array}{l}\text { MACE in patients } \\
\text { with mild or } \\
\text { no LUTS, \% }\end{array}$ & NOS score & MACE definition \\
\hline $\begin{array}{l}\text { Wehrberger } \\
\text { et al, } 2011[10]\end{array}$ & $\mathrm{CB} / \mathrm{MACE}$ incidence & 2092 & 47.8 & 73 & 354 & 5.6 & 1738 & 4.4 & 8 & ICD codes: I20-25, I60-65 \\
\hline Lin et al, 2013 [22] & CB/MACE incidence & $11748^{*}$ & 47 & 80 & 1958 & 5.3 & 9790 & 3.5 & 7 & $\begin{array}{l}\text { ICD-9 codes: } 410,411,430 \text {, } \\
431,433-436\end{array}$ \\
\hline $\begin{array}{l}\text { Bouwman } \\
\text { et al, } 2014 \text { [23] }\end{array}$ & $\mathrm{CB} / \mathrm{MACE}$ incidence & 1246 & 61.4 & 103 & 267 & 4.5 & 979 & 4.7 & 7 & $\begin{array}{l}\text { ICPC codes: K74, K75, K76, } \\
\text { K89, K90 }\end{array}$ \\
\hline $\begin{array}{l}\text { Bouwman } \\
\text { et al, } 2014[21]^{\S}\end{array}$ & PCP/MACE incidence & 6614 & 56 & 120 & 1165 & 41.1 & 5449 & 19.5 & 8 & $\begin{array}{l}\text { ATC definitions for AMI, } \\
\text { stroke, sudden death }\end{array}$ \\
\hline $\begin{array}{l}\text { Kupelian } \\
\text { et al, } 2015 \text { [24] }\end{array}$ & $\mathrm{CB} / \mathrm{MACE}$ incidence & 3794 & 50.2 & 58 & 695 & 7.9 & 3099 & 4.4 & 8 & ICPC codes: K74, K75, K76, K77 \\
\hline $\begin{array}{l}\text { Weisman } \\
\text { et al, } 2000[13]\end{array}$ & UP/MACE prevalence & 140 & 72 & 24 & 108 & 28.7 & 32 & 9.3 & 6 & ICPC codes: K75, K76 \\
\hline Meigs et al, 2001 [9] & $\mathrm{CB} / \mathrm{MACE}$ prevalence & 1019 & 53.9 & 36 & 198 & 18.7 & 821 & 7.8 & 8 & ICPC codes: K75, K76 \\
\hline Seim et al, 2005 [14] & $\mathrm{CB} / \mathrm{MACE}$ prevalence & 21627 & 58.9 & 24 & 3410 & 4.2 & 18217 & 1.3 & 8 & ICPC codes: K75, K76, K90 \\
\hline Wong et al, 2006 [15] & $\mathrm{CB} / \mathrm{MACE}$ prevalence & 1737 & 72.9 & - & 665 & 19.3 & 1072 & 12.8 & 7 & ICPC codes: K75, K76 \\
\hline El-Sakka, 2006 [16] & UP/MACE prevalence & 476 & 51.8 & 30 & 369 & 26.6 & 107 & 3.7 & 7 & ICPC codes: K75, K76 \\
\hline Coyne et al, 2009 [17] & $\mathrm{CB} / \mathrm{MACE}$ prevalence & $7530^{*}$ & 56.6 & 10 & 3433 & 16 & 4097 & 5 & 8 & ICPC codes: K75, K76 \\
\hline $\begin{array}{l}\text { Kupelian } \\
\quad \text { et al, } 2009 \text { [18] }\end{array}$ & $\mathrm{CB} / \mathrm{MACE}$ prevalence & $2301^{*}$ & - & 36 & 441 & 22.3 & 1860 & 7.9 & 8 & ICPC codes: K74, K75, K76, K77 \\
\hline Wong et al, 2010 [19] & $\mathrm{CB} / \mathrm{MACE}$ prevalence & 871 & 71.3 & 48 & 194 & 17.5 & 677 & 11.2 & 7 & ICPC codes: K75, K76 \\
\hline Barbosa et al, 2013 [20] & UP/MACE prevalence & 907 & 61 & 12 & 499 & 22.4 & 408 & 10.3 & 7 & ICPC codes: K75, K76 \\
\hline $\begin{array}{l}\text { Bouwman } \\
\text { et al, } 2014[21]^{\S}\end{array}$ & $\mathrm{CB} / \mathrm{MACE}$ prevalence & 1610 & 61.4 & 103 & 392 & 32.6 & 1218 & 18.9 & 7 & $\begin{array}{l}\text { ATC definitions for AMI, } \\
\text { stroke, sudden death }\end{array}$ \\
\hline \multicolumn{11}{|c|}{$\begin{array}{l}\text { AMI = acute myocardial infarction; ATC = Antithrombotic Trialists Collaboration; } \mathrm{CB}=\text { community-based; ICD }=\text { International Classification of Diseases; ICPC = International Classification of Primary Care; LUTS = lower } \\
\text { urinary tract symptoms; MACE = major adverse cardiac events; NOS = Newcastle-Ottawa quality assessment scale; PCP = primary care population; UP = urology practice. } \\
\text { The following ICPC codes were used: K74, angina pectoris; K75, AMI; K76, other/chronic coronary heart disease; K77, congestive heart failure; K89, transient cerebral ischemia; K90, cerebrovascular accident. } \\
\text { Male cohort extrapolated from the study population. }\end{array}$} \\
\hline
\end{tabular}


prostatic hyperplasia (BPH) and/or lower urinary tract symptoms (LUTS) and cardiovascular, cardio, major adverse cardiac events, MACE, heart disease, heart, myocardial infarction, myocardial, infarction, stroke, ischemic events, ischemic, cardiac death, coronary syndrome. The search through September 30, 2015, was restricted to Englishlanguage articles and studies of human participants. Bibliographies of retrieved papers were hand searched for additional references. Details of the literature search process are outlined in the flow chart. Identification of relevant abstracts, selection of studies based on the criteria described, and subsequent data extraction were performed independently by two authors (A.S., M.G.), and conflicts were resolved by a third investigator (G.C.). Full-text articles and meeting abstracts were included.

\subsection{Study selection}

We included all cross-sectional and longitudinal trials enrolling men and comparing the prevalence or incidence of MACE in patients with moderate to severe LUTS compared with those with mild or without LUTS [9,10,13-24] (Fig. 1 and Table 1). Studies not specifically stating the occurrence or absence of cardiovascular-related events or the severity of LUTS were excluded from the analysis. In addition, articles in which only nocturia was evaluated were excluded from the analysis [25]. One study was excluded because only the OR was reported, not allowing proper data analysis.

\subsection{Outcome and quality assessment}

The principal outcome of this analysis was to evaluate the incidence of MACE in patients with moderate to severe LUTS in comparison to those with mild or no LUTS. A secondary outcome included the prevalence of MACE in participants with the same characteristics. The quality of the studies was assessed using the Cochrane criteria [26].

\subsection{Statistical analysis}

Heterogeneity of prevalence of MACE in patients with moderate to severe LUTS in comparison to those with mild or no LUTS was assessed using $\mathrm{I}^{2}$ statistics. Even when low heterogeneity was detected, a random-effects model was applied because the validity of tests of heterogeneity can be limited with a small number of component studies. To estimate possible publication or disclosure bias, we used funnel plots, the Begg adjusted rank correlation test, and Egger's test. A metaregression analysis was performed to test the effect of age and associated morbidities (including diabetes and hypertension) on MACE prevalence. In addition, a linear regression analysis model weighting each study for the number of participants enrolled was carried out to verify the independent effect of diabetes on LUTSrelated MACE after adjustment for age. All data were calculated using Comprehensive Meta-Analysis software version 2 (Biostat, Englewood, NJ, USA). Logistic multivariate analysis was done in SPSS for Windows 20.1 (IBM Corp, Armonk, NY, USA).

\section{Evidence synthesis}

\subsection{Results}

Of 477 retrieved articles, 463 articles were excluded for different reasons. The flow of the meta-analysis is summarized in Fig. 1, and the characteristics of the trials included in the meta-analysis are summarized in Table $1[9,10$, 13-24]. Thirty-one full-text articles were considered as potentially eligible for the meta-analysis; however, 17 of them were excluded because they did not include clear information about MACE or LUTS.

Among the 14 selected studies, 10 studies [9,13-21] reported the prevalence of history of MACE at enrollment in patients with moderate to severe LUTS compared with those with mild or no LUTS (Table 1). In addition, five trials longitudinally collected the incidence of MACE in participants with the same characteristics (Table 1) [10,21-24].One of the trials [21] reported both the prevalence of MACE in the populations of interest and the incidence of MACE after the exclusion of men with a history of CVD.

Among the cross-sectional studies, 38218 patients and 2527 MACE were included in the meta-analysis. The mean age of enrolled patients was $62.2 \pm 8.0 \mathrm{yr}$. In addition, the mean prevalence of diabetes and hypertension was $18.6 \%$ and $33.8 \%$, respectively. $\mathrm{I}^{2}$ in cross-sectional trials was 79.9 $(p<0.0001)$. The funnel plot and Begg adjusted rank correlation test (Kendall $\tau: 0.0 ; p=1.0$ ) suggested no major publication bias. The presence of moderate to severe LUTS is significantly associated with an increased prevalence of reported history of MACE (OR: 2.38; 95\% confidence interval [CI], 2.56-3.07; $p<0.001$ ) (Fig. 2).

Metaregression analysis showed that the risk of MACE was lower in older patients and higher in participants with reported higher prevalence of diabetes (Fig. 3). Accordingly, a higher risk of LUTS-associated MACE was detected in those studies enrolling participants with a mean age $<60 \mathrm{yr}$ at enrollment (OR: 3.40 [95\% CI, 2.78-4.17] vs 1.96 [95\% CI, 1.62-3.37]; $Q=15.12 ; p<0.0001)$. A linear regression analysis model weighting each study for the number of participants enrolled was carried out to verify the independent effect of diabetes on moderate/severe LUTSassociated MACE. When age and diabetes were introduced as possible confounders and LUTS-related MACE was considered as a dependent variable, diabetes was significantly associated with MACE, even after adjustment for age (adjusted $r=0.498 ; p<0.0001$ ). Similar results were observed when trial follow-up was introduced in the model as a possible further confounder (adjusted $r=0.478$; $p<0.0001)$. Conversely, no relationship between MACE and hypertension was observed (data not shown).

Longitudinal trials included 25494 patients and 2291 MACE. The mean age of enrolled patients was $52.5 \pm 5.5 \mathrm{yr}$, and the mean follow-up was $86.8 \pm 22.1 \mathrm{mo}$. The presence of moderate to severe LUTS was associated with a significantly increased incidence of MACE compared with the rest of the sample (OR: $1.68 ; 95 \% \mathrm{CI}, 1.13-2.50 ; p=0.01$ ) (Fig. 4). 


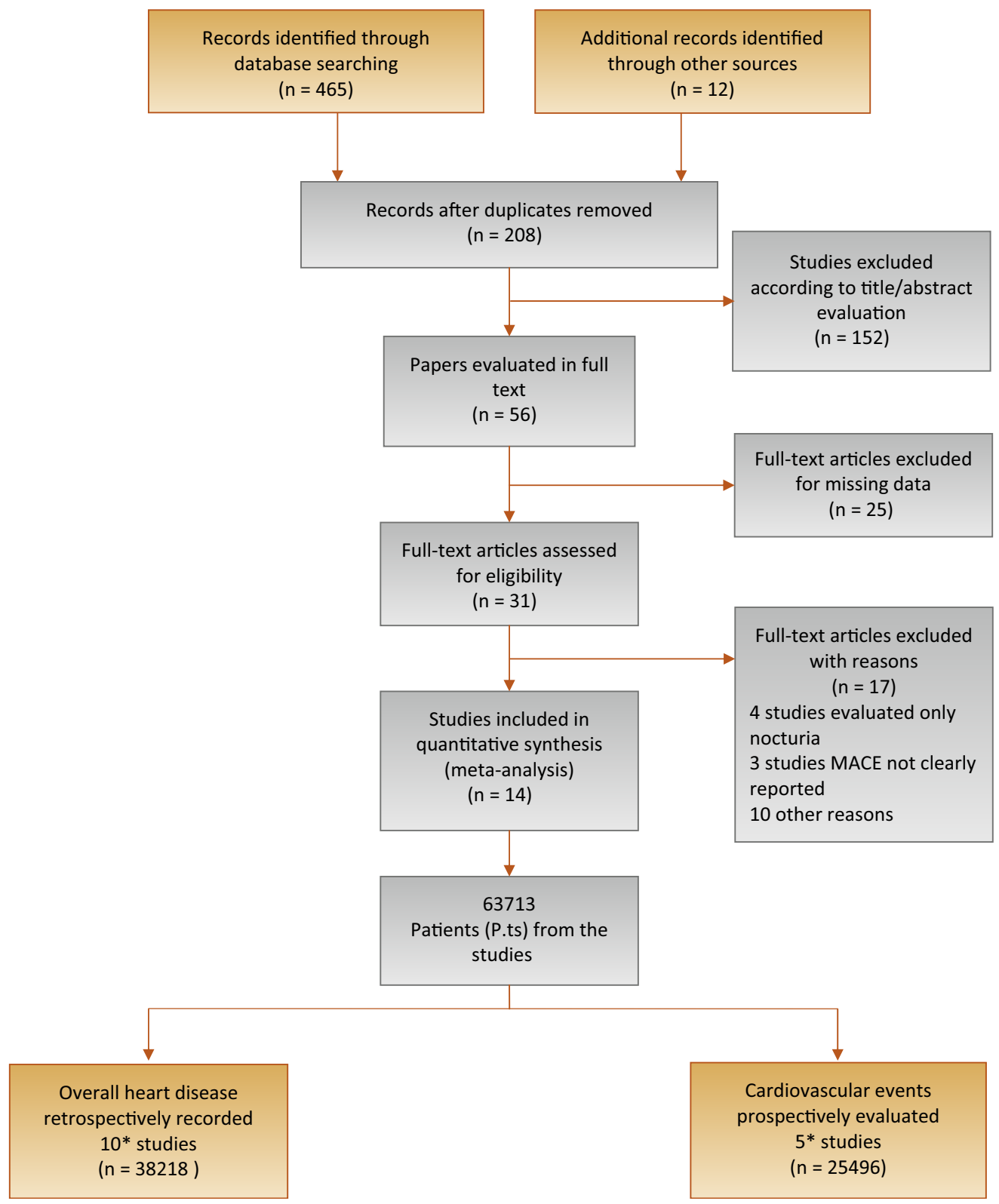

Fig. 1 - Flow diagram of literature searches according to the Preferred Reporting Items for Systematic Reviews and Meta-Analyses statement. MACE $=$ major adverse cardiac events.

${ }^{*}$ One of the trials [21] reported both the prevalence of MACE in the populations of interest and the incidence of MACE after the exclusion of men with a history of cardiovascular disease.

\subsection{Discussion}

MACE and LUTS are health problems that are becoming more prevalent in the worldwide aging population. The association between CVD and micturition disorders has been investigated previously in white, black, and Chinese populations [19]. In the present meta-analytic systematic review, we showed that patients with moderate to severe LUTS have an increased risk of MACE, most probably because the two conditions share similar determinants. In particular, men with metabolic conditions, including diabetes, may present with alterations in vascular supply and innervation of several tissues (which may include bladder and prostate), finally leading to increased cardiovascular risk and MACE
[27]. Accordingly, the present data indicated that the risk of LUTS-associated MACE increased as a function of diabetes prevalence.

In the EpiLUTS study, heart disease was independently associated with voiding symptoms combined with storage and postmicturition symptoms, suggesting that LUTS and MACE could share common pathophysiologic pathways [17]. MetS represents a constellation of pathologic conditions (hypertension, obesity, hyperglycemia, and dyslipidemia), reflecting insulin resistance and association with increased cardiovascular and metabolic risk [28]. Accordingly, in a meta-analysis of available evidence, Mottillo et al [8] reported a twofold increase in the risk of CVD in men with MetS. Interestingly, we previously reported that MetS was 


\begin{tabular}{|c|c|c|c|c|c|c|c|c|}
\hline \multirow[t]{2}{*}{ Study name } & \multicolumn{4}{|c|}{ Statistics for each study } & \multicolumn{4}{|c|}{ Odds ratio and $95 \% \mathrm{Cl}$} \\
\hline & $\begin{array}{l}\text { Odds } \\
\text { ratio }\end{array}$ & $\begin{array}{l}\text { Lower } \\
\text { limit }\end{array}$ & $\begin{array}{l}\text { Upper } \\
\text { limit }\end{array}$ & $p$ value & & & & \\
\hline Weisman et al, 2000 & 3.89 & 1.10 & 13.72 & 0.03 & & & $\longrightarrow+$ & \\
\hline Meigs et al, 2001 & 2.72 & 1.75 & 4.22 & 0.00 & & & $\square$ & \\
\hline Seim et al, 2005 & 3.18 & 2.58 & 3.91 & 0.00 & & & $\square$ & \\
\hline Wong et al, 2006 & 1.63 & 1.25 & 2.12 & 0.00 & & & $\square$ & \\
\hline El Sakka et al, 2006 & 9.31 & 3.34 & 25.96 & 0.00 & & & & \\
\hline Coyne et al, 2009 & 3.59 & 3.04 & 4.25 & 0.00 & & & $\square$ & \\
\hline Kupelian et al, 2009 & 3.46 & 2.62 & 4.57 & 0.00 & & & $\square$ & \\
\hline Wong et al, 2009 & 1.68 & 1.08 & 2.61 & 0.02 & & & $\rightarrow$ & \\
\hline Barbosa et al, 2013 & 2.52 & 1.72 & 3.70 & 0.00 & & & $\rightarrow-$ & \\
\hline Bouwman et al, 2014 & 2.07 & 1.61 & 2.67 & 0.00 & & & $\square$ & \\
\hline \multirow[t]{3}{*}{ Overall } & 2.80 & 2.56 & 3.07 & 0.00 & & & 1 & \\
\hline & & & & & 0.01 & 0.1 & 10 & 100 \\
\hline & \multicolumn{8}{|c|}{ No Mild } \\
\hline
\end{tabular}

Fig. 2 - Risk (odds ratio) of reporting a history of major adverse cardiac events according to lower urinary tract symptom status (no/mild vs moderate/ severe). Presentation in order of publication year.

CI = confidence interval; LUTS = lower urinary tract symptoms.
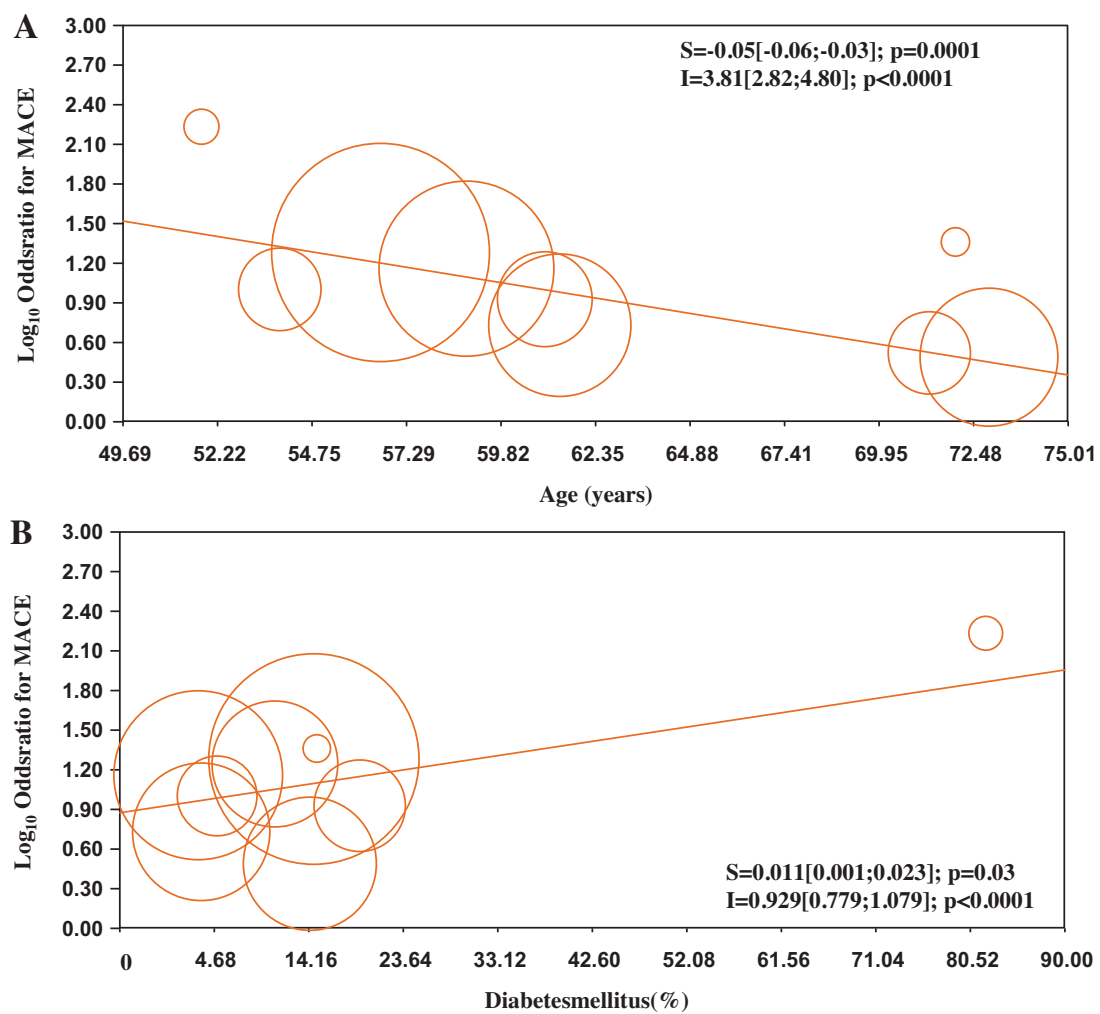

Fig. 3 - Influence of (a) age and (b) diabetes mellitus on the risk of major adverse cardiac events. MACE = major adverse cardiac events; $S$ = slope; $I=$ intercept.

associated with a larger total and transitional prostatic volume, confirming a pathologic link between MetS and $\mathrm{BPH}$ [6]. In this review, we showed that $\mathrm{BPH}$ is significantly associated with higher cardiovascular risk tightly related to the prevalence of diabetes mellitus. The latter association was confirmed even when adjusted for age, suggesting that $\mathrm{BPH}$ is an early marker of cardiovascular risk rather than playing a direct pathogenic role in cardiovascular risk stratification. Accordingly, the risk was lower in older participants (particularly those aged $>60 \mathrm{yr}$ ), for whom other major conventional cardiovascular risk might smooth the significance of LUTS. Our data are in line with the findings of the Boston Area Community Health (BACH) population-based epidemiological survey of 


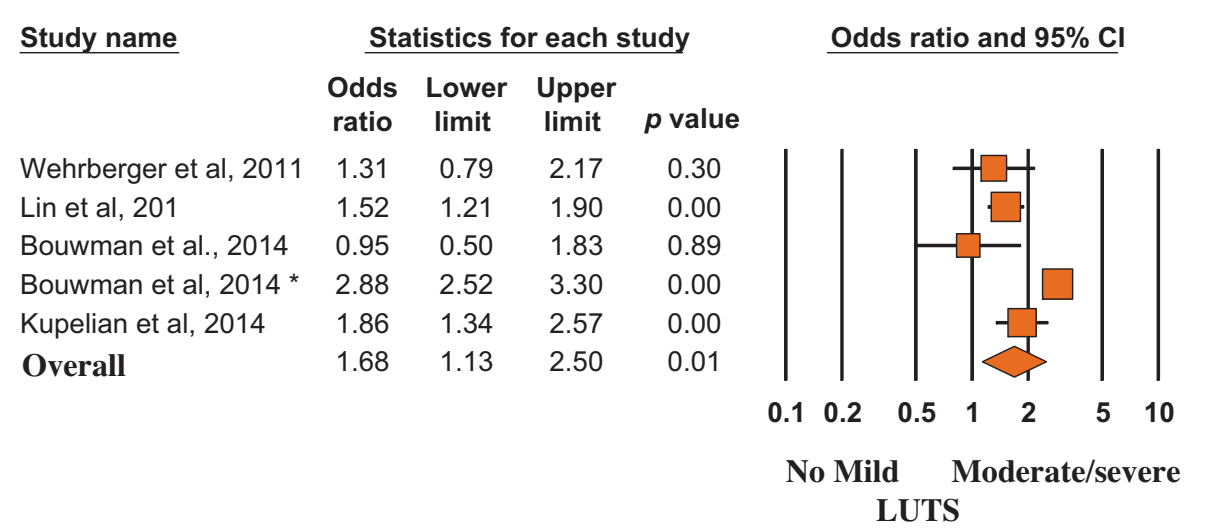

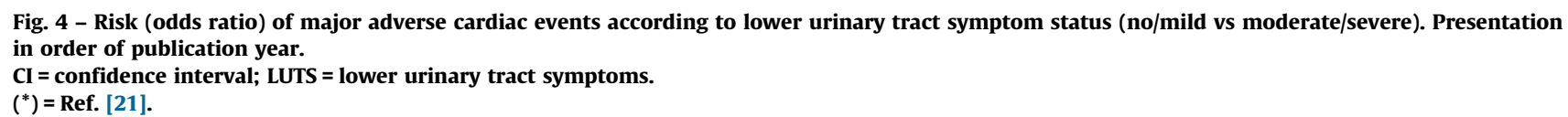

urologic symptoms [18,24,29], showing that, after a median follow-up of $4.8 \mathrm{yr}$, LUTS was associated with earlier diabetes onset in younger men (aged $<50 \mathrm{yr}$ ). The association of moderate to severe LUTS with diabetes was evident only in men, not in women, and the increased risk of diabetes and heart disease occurred in obese LUTS patients, suggesting that LUTS could be considered as a sentinel marker for diabetes and heart disease.

In a recent study of MetS and prostate abnormalities in young men (mean age: $36 \mathrm{yr}$ ) of infertile couples, Lotti et al [30] reported that MetS was positively associated not only with biochemical (seminal interleukin 8) and ultrasoundderived signs of prostatic inflammation but also with prostate enlargement, suggesting that MetS could be a determinant of an early onset form of BPE, as a possible response of prostatic tissue to a chronic inflammation. The same authors reported that insulin levels were associated with prostate volume even after adjustment for confounders [30], suggesting that prostate overgrowth in young men may be due to a hyperinsulinemic state leading to chronic prostatic inflammation.

Although historically considered a "normal" consequence of the aging process, LUTS/BPH should be faced proactively as a preventable disorder in elderly patients. Type of diet and level of physical activity are emerging as other important factors affecting prostate health in aging men, most probably reducing risk factors such as MetS, hypogonadism, and inflammation [31,32]. Whether physical exercise, weight loss, and modifications of dietary habit can really alter the natural history of LUTS/BPH remains to be determined.

In a meta-analysis limited to five longitudinal trials, including 6027 men with LUTS and 18993 men without LUTS, Bouwman et al [33] failed to find an association between LUTS severity and CVD. Some important differences in trial selection compared with the present study must be emphasized. Bouwman et al [33] excluded one trial [24] because the authors applied logistic regression analyses instead of survival analyses, whereas they included a trial targeted exclusively at nocturia [25] that was excluded from our analysis. We excluded four trials on nocturia from our review (Fig. 1) because nocturia can be caused by a variety of other factors, including aberrant behavior (fluid intake), systemic disorders (respiratory, neurogenic, endocrine, nephrologic), sleep disorders, and impaired storage function. Moreover, because lower urinary tract dysfunction is the cause of nocturia in only a minority of cases, it may be more appropriate to define nocturia as a systemic symptom rather than as LUTS [34]. Study selection, adjustment for different items, statistical methods, and different LUTS and MACE definitions could cause different outcomes between the reviews.

The findings of our review may have clinical implications (1) for prevention, by redefining the role of lifestyle (diet and physical activity) for men at risk of both urinary symptoms and heart disease; (2) for diagnosis, by suggesting screening for CVD in men with LUTS and vice versa; and (3) for treatment, by promoting a holistic approach (general practitioner, urologist, endocrinologist, cardiologist) to treat men with comorbid MetS, LUTS, and CVD. The main limitation of our review is surveillance bias, as men with LUTS or MACE might be more likely to seek health care and receive medical diagnoses. Moreover, none of the trials included in the review estimated the impact of behavioral education and medical treatment for MACE on LUTS and vice versa. Furthermore, our data were not adjusted for some relevant issues such as family history (of both LUTS and MACE), diet, smoking, physical activity, and pertinent comorbidities including depression. Finally, we were not able to include some other relevant urologic parameters, such as prostate volume, uroflowmetry, or postvoid residual volume, in the analysis.

\section{Conclusions}

We reported that moderate to severe LUTS can be considered as a sentinel marker for diabetes and heart 
disease. Further longitudinal trials are needed to confirm these data. Our message is, "Take prostatic health to heart!"

Author contributions: Mauro Gacci had full access to all the data in the study and takes responsibility for the integrity of the data and the accuracy of the data analysis.

Study concept and design: Gacci.

Acquisition of data: Sebastianelli, Serni.

Analysis and interpretation of data: Gacci, Maggi, De Nunzio, Novara.

Drafting of the manuscript: Gacci, Maggi, De Nunzio, Novara.

Critical revision of the manuscript for important intellectual content:

McVary, Kaplan, Gravas, Chapple.

Statistical analysis: Corona, Vignozzi.

Obtaining funding: None.

Administrative, technical, or material support: None.

Supervision: Gacci.

Other (specify): Giovanni Corona and Mauro Gacci equally contributed to this work.

Financial disclosures: Mauro Gacci certifies that all conflicts of interest, including specific financial interests and relationships and affiliations relevant to the subject matter or materials discussed in the manuscript (eg, employment/affiliation, grants or funding, consultancies, honoraria, stock ownership or options, expert testimony, royalties, or patents filed, received, or pending), are the following: None.

Funding/Support and role of the sponsor: None.

\section{Appendix A. Supplementary data}

Supplementary material related to this article can be found, in the online version, at http://dx.doi.org/10.1016/j. eururo.2016.07.007.

\section{References}

[1] Richard F, Lukacs B, Jardin A, et al. Results of an epidemiologic surgery carried out with men 50-80 years of age to study urinary disorders, quality of life and sexual function. Prog Urol 2001;11: 250-63.

[2] Verhamme KMC, Dieleman JP, Bleumink GS, Van derLei J, Sturkenboom MCJM. TRIUMPH Pan European Expert Panel. Incidence and prevalence of lower urinary tract symptoms suggestive of benign prostatic hyperpklasia in primary care - the TRIUMPH project. Eur Urol 2002;42:323-8.

[3] McDonnell J, Busschbach JJ, Kok E, et al. Lower urinary tract symptoms suggestive of benign prostatic obstruction-Triumph: healtheconomical analysis. Eur Urol 2001;39(Suppl 3):37-41.

[4] Gratzke C, Bachmann A, Descazeaud A, et al. EAU guidelines on the assessment of non-neurogenic male lower urinary tract symptoms including benign prostatic obstruction. Eur Urol 2015;67:1099-109.

[5] Vignozzi L, Gacci M, Cellai I, et al. Fat boosts, while androgen receptor activation counteracts, BPH-associated prostate inflammation. Prostate 2013;73:789-800.

[6] Gacci M, Corona G, Vignozzi L, et al. Metabolic syndrome and benign prostatic enlargement: a systematic review and meta-analysis. BJU Int 2015;115:24-31.

[7] Kahn R, Buse J, Ferrannini E, Stern M. The metabolic syndrome: time for a critical appraisal. Joint statement from the American Diabetes Association and the European Association for the Study of Diabetes. Diabetologia 2005;48:1684-99.
[8] Mottillo S, Filion KB, Genest J, et al. The metabolic syndrome and cardiovascular risk a systematic review and meta-analysis. J Am Coll Cardiol 2010;56:1113-32.

[9] Meigs JB, Mohr B, Barry MJ, Collins MM, McKinlay JB. Risk factors for clinical benign prostatic hyperplasia in a community-based population of healthy aging men. J Clin Epidemiol 2001;54:935-44.

[10] Wehrberger C, Temml C, Gutjahr G, et al. Is there an association between lower urinary tract symptoms and cardiovascular risk in men? A cross sectional and longitudinal analysis. Urology 2011;78: 1063-7.

[11] Stroup DF, Berlin JA, Morton SC, et al. Meta-analysis of observational studies in epidemiology: a proposal for reporting. Metaanalysis Of Observational Studies in Epidemiology (MOOSE) group. JAMA 2000;283:2008-12.

[12] International Classification of Primary Care, ed 2 (ICPC-2). Geneva, Switzerland: World Health Organization; 2003.

[13] Weisman KM, Larijani GE, Goldstein MR, Goldberg ME. Relationship between benign prostatic hyperplasia and history of coronary artery disease in elderly men. Pharmacotherapy 2000;20:383-6.

[14] Seim A, Hoyo C, Ostbye T, Vatten L. The prevalence and correlates of urinary tract symptoms in Norwegian men: the HUNT study. BJU Int 2005;96:88-92.

[15] Wong SY, Woo J, Hong A, Leung JC, Kwok T, Leung PC. Risk factors for lower urinary tract symptoms in southern Chinese men. Urology 2006;68:1009-14.

[16] El-Sakka AI. Lower urinary tract symptoms in patients with erectile dysfunction: analysis of risk factors. J Sex Med 2006;3:144-9.

[17] Coyne KS, Kaplan SA, Chapple CR, et al. EpiLUTS Team. Risk factors and comorbid conditions associated with lower urinary tract symptoms: EpiLUTS. BJU Int 2009;103(Suppl 3):24-32.

[18] Kupelian V, Rosen RC, Link CL, et al. Association of urological symptoms and chronic illness in men and women: contributions of symptom severity and duration-results from the BACH Survey. J Urol 2009;181:694-700.

[19] Wong SY, Woo J, Leung JC, Leung PC. Depressive symptoms and lifestyle factors as risk factors of lower urinary tract symptoms in Southern Chinese men: a prospective study. Aging Male 2010;13: 113-9.

[20] Barbosa JA, Muracca E, Nakano E, et al. Risk factors for male lower urinary tract symptoms: the role of metabolic syndrome and androgenetic alopecia in a Latin American population. Urology 2013;82:182-8.

[21] Bouwman II, Blanker MH, Schouten BW, et al. Are lower urinary tract symptoms associated with cardiovascular disease in the Dutch general population? Results from the Krimpen study. World J Urol 2015;33:669-76.

[22] Lin HJ, Weng SF, Yang CM, Wu MP. Risk of hospitalization for acute cardiovascular events among subjects with lower urinary tract symptoms: a nationwide population-based study. PLoS One 2013;8:e66661.

[23] Bouwman II, Kollen BJ, van der Meer K, Nijman RJ, van der Heide WK. Are lower urinary tract symptoms in men associated with cardiovascular diseases in a primary care population: a registry study. BMC Fam Pract 2014;15:9.

[24] Kupelian V, Araujo AB, Wittert GA, McKinlay JB. Association of moderate to severe lower urinary tract symptoms with incident type 2 diabetes and heart disease. J Urol 2015;193:581-6.

[25] Lightner DJ, Krambeck AE, Jacobson DJ, et al. Nocturia is associated with an increased risk of coronary heart disease and death. BJU Int 2012;110:848-53.

[26] Higgins JP1, Altman DG, Gøtzsche PC, et al. Cochrane Bias Methods Group, Cochrane Statistical Methods Group. The Cochrane Collaboration's tool for assessing risk of bias in randomised trials. BMJ 2011;343:d5928. 
[27] Gacci M, Eardley I, Giuliano F, et al. Critical analysis of the relationship between sexual dysfunctions and lower urinary tract symptoms due to benign prostatic hyperplasia. Eur Urol 2011;60:809-25.

[28] Corona G, Monami M, Rastrelli G, et al. Is metabolic syndrome a useless category in subjects with high cardiovascular risk? Results from a cohort study in men with erectile dysfunction. J Sex Med 2011;8:504-11.

[29] Kupelian V, McVary KT, Kaplan SA, et al. Association of lower urinary tract symptoms and the metabolic syndrome: results from the Boston Area Community Health Survey. J Urol 2009;182:616-24.

[30] Lotti F, Corona G, Vignozzi L, et al. Metabolic syndrome and prostate abnormalities in male subjects of infertile couples. Asian J Androl 2014;16:295-304.
[31] Corona G, Vignozzi L, Rastrelli G, Lotti F, Cipriani S, Maggi M. Benign prostatic hyperplasia a new metabolic disease of the aging male and its correlates with sexual dysfunctions. Int J Endocrinol 2014;2014:329456.

[32] Vignozzi L, Rastrelli G, Corona G, Gacci M, Forti G, Maggi M. Being prostatic hyperplasia: a new metabolic disease? J Endocrinol Invest 2014;37:313-22.

[33] Bouwman II, Voskamp MJ, Kollen BJ, Nijman RJ, van der Heide WK, Blanker MH. Do lower urinary tract symptoms predict cardiovascular diseases in older men? A systematic review and meta-analysis. World J Urol 2015;33:1911-20.

[34] Drake MJ. Should nocturia not be called a lower urinary tract symptom? Eur Urol 2015;67:289-90.

www.esusalzburg17.org

\title{
ESU - Weill Cornell Masterclass
} in General urology

\author{
9-15 July 2017, Salzburg, Austria
}

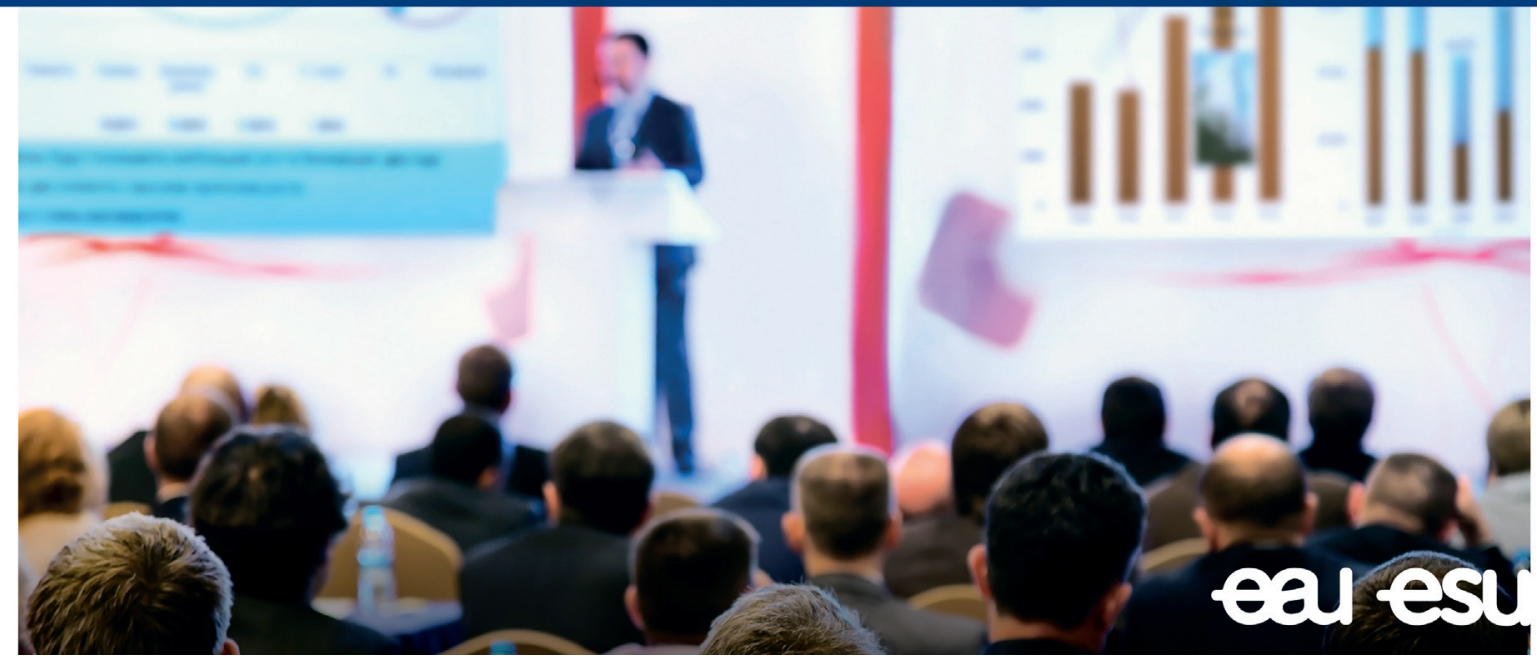

EAU Events

are accredited

by the EBU in

the UEMS/EACCME

regulations 